\title{
High-Performance Navigation System with Integration of Low-Precision MEMS INS and General-Purpose GPS
}

\author{
By Masaru NARUOKA and Takeshi TsuchiYA \\ Department of Aeronautics and Astronautics, The University of Tokyo, Tokyo, Japan
}

(Received February 21st, 2007)

\begin{abstract}
In this paper, we propose a new configuration for a strap-down, integrated INS/GPS (Inertial Navigation System/ Global Positioning System) navigation system. It is aimed at general-purpose use, and utilizes MEMS (microelectromechanical system) sensors and a quaternion-based model that enable the development of a precise system with a degree of portability that traditional INS/GPS devices cannot achieve. In order to examine the effectiveness of our system, we built a prototype instrument and performed an experiment comparing its performance with GAIA, an ultra highprecision INS/GPS device developed by the Japan Aerospace Exploration Agency (JAXA). The results show that our small, light and low-cost system is applicable to general-purpose use, having a position error of only a few meters and under 2 degrees of roll and pitch error, which is sufficiently precise for the general control of moving objects.
\end{abstract}

Key Words: Navigation, INS, GPS, MEMS

\section{Nomenclature}

$\underline{x}$ : general column vector

$\vec{x}$ : general three-dimensional vector

$\tilde{q}$ : general quaternion

$\tilde{q}^{*}$ : general conjugate quaternion

$\vec{r}_{e}$ : target position vector from the center of the Earth

$\overrightarrow{\vec{r}}_{e}^{n}$ : target velocity to the Earth (3 states)

$\vec{a}^{b}$ : output of accelerometers which are fixed on the target (3 states)

$\vec{\omega}_{e / i}^{e}$ : Earth rotation on Earth-Centered Earth-Fixed frame (3 states)

$\vec{\omega}_{n / e}^{n}$ : navigation frame rotation (3 states)

$\vec{\omega}_{b / i}^{b}$ : output of gyros which are fixed on the target (3 states)

$\vec{g}^{n}$ : Earth gravitation (3 states)

$h$ : altitude (1 state)

$\tilde{q}_{e}^{n}$ : position quaternion of latitude, longitude, and azimuth (4 states)

$\tilde{q}_{n}^{b}:$ attitude quaternion of roll, pitch, and yaw (4 states)

$P$ : system error covariance matrix of Kalman Filter

$Q$ : process error covariance matrix of Kalman Filter

$R$ : measurement error covariance matrix of Kalman Filter

$K$ : Kalman gain

\section{Introduction}

Our study is a response to the current need for controlling or monitoring moving objects such as vehicles, robots and so on. Strap-down INS/GPS systems have great potential to serve in these applications because such systems give precise state values: position, velocity, and attitude of the moving objects. However, traditional INS/GPS devices are only rarely installed into these moving objects. Most devices of

(C) 2008 The Japan Society for Aeronautical and Space Sciences this type are used in aircraft and spacecraft which require highly precise navigation, and are unsuitable for other purposes due to their lack of portability and general high cost. Such systems are large, heavy, and expensive dedicated devices.

Therefore, we have developed a new strap-down INS/ GPS configuration that is small, light, and low-cost. This configuration is characterized by two features. First is the use of MEMS inertial sensors and a civil-use GPS receiver as INS/GPS components. They are low-cost, small, and light weight. However, MEMS sensors do not have a level of precision sufficient for navigation because of their low signal-to-noise $(\mathrm{S} / \mathrm{N})$ ratio. Winkler et al. ${ }^{1)}$ and Liu et al. ${ }^{2}$ reported INS/GPS configurations that employed a similar concept and overcame the problem by calibrating the sensors and so on. However, we do not consider such ideas sufficiently robust. Thus, we introduce another feature into the system.

This feature is quaternion modeling for extended Kalman filtering (EKF), by which an INS and a GPS are integrated and function as an INS/GPS. It is noted that applying EKF to nonlinear system models that have strong nonlinearity like singular points often causes divergence because their linearized models in the EKF do not reflect real models accurately. To decrease this possibility of divergence, we make a new algorithm that removes singular points and simplifies the models mathematically by using quaternions. In addition, using quaternions is superior to using direction cosine matrices (DCMs), which is a well-known method to elude singular points, in computational costs (i.e., the number of variables of a quaternion is 4 and that of a DCM is 6).

In order to examine the effectiveness of our configuration, we built a prototype system and tested it experimentally. The main components of the prototype system are tri-axes MEMS accelerometers, tri-axes MEMS gyros, a L1-wave 
GPS receiver module, and a data processing PC. After we calibrated its temperature characteristics and misalignment, the experiment for comparing the prototype with the GAIA, ${ }^{3)}$ a high-precision INS/GPS instrument developed by the Japan Aerospace Exploration Agency (JAXA), was performed to evaluate the precision of the proposed system during the flight of an experimental aircraft, the MuPAL- $\alpha$ of JAXA.

\section{INS/GPS Components}

In this section, we describe the components of the INS/ GPS device we configured. The INS/GPS device consists of an INS and a GPS as physical components; therefore, we explain these two components in sequence. The two components are integrated as an INS/GPS integrated system by our algorithm as described in the next section.

\subsection{Strap-down INS using MEMS sensors}

An INS outputs position, velocity, and attitude by obtaining acceleration and angular speed from inertial sensors and solving kinematic equations. According to the method of mounting the inertial sensors, INSs are classified roughly into two types: strap-down type and gimbaled type. Although it needs relatively large calculation power, the strap-down type does not require physical mechanisms and can therefore be configured as a smaller, lighter, and lower-cost INS. For these reasons we adopted the strap-down type.

Characteristics of an INS such as precision, and cost are derived mostly from installed inertial sensors. In our configuration, MEMS sensors which are widely used for civil-use products such as car navigation systems are chosen. They are much smaller, lighter, and less expensive than high-precision sensors such as servo accelerometers and ring laser gyros, which are intended for aircraft and spacecraft navigation. However, they have low $\mathrm{S} / \mathrm{N}$ (signal-to-noise) ratios and low bias stability, and they vary considerably in quality. Moreover, it is noted that the precision of the INS which employs them is very low. In order to compensate for that disadvantage, we take advantage of integration with a GPS.

\subsection{Civil-use GPS receiver}

The function of GPS ground nodes is estimating position, velocity, and so on by receiving radio waves emitted by GPS satellites. Therefore, the characteristics of a GPS such as precision and cost depend on the type of receiver and antenna used. Specifically, these element are related to the system's data processing method and the frequency bands employed. We select a receiver and antenna which receive the GPS L1 frequency band (1575 MHz) and perform C/A code stand-alone measurement. They are commonly used, for example, in car navigation, and are small, light weight, and inexpensive.

\section{INS/GPS Algorithm}

In this section, we explain the INS/GPS algorithm we configured. First, the coordinate systems and notations we use are described. We then explain the extended Kalman fil-

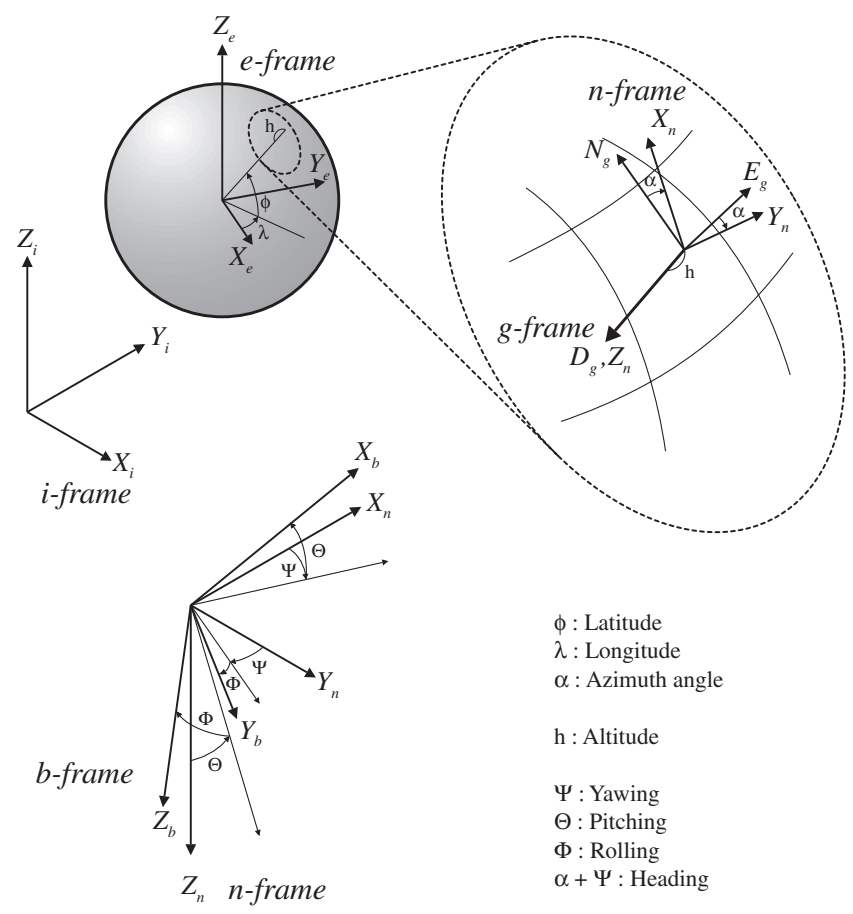

Fig. 1. Coordinate systems.

ter (EKF) that is the base of our algorithm, and derive the system equation and the measurement equation that are required to apply the filter. Finally, we describe the algorithm based on two stages (i.e., time update and measurement update) that are defined in the filter, and show the performance of our algorithm.

\subsection{Coordinate systems and symbol definitions}

We use five coordinate systems as shown in Fig. 1. All systems are right-handed orthogonal systems.

- $i$-Frame: The Earth-centered inertial system. The $Z_{i}$ axis corresponds to the rotation axis of the Earth.

- e-Frame: The Earth-centered, Earth-fixed system. The $Z_{i}$ axis corresponds to the rotation axis of the Earth, and the $X_{e}$ axis is directed to the first meridian.

- g-Frame: The local geodetic system. The origin is the observation point. The $N_{g}$ axis and $D_{g}$ axis are directed northward and downward, respectively.

- $n$-Frame: The navigation frame. This system is identical to the rotated g-Frame by Azimuth angle, $\alpha$ rad.

- b-Frame: The body frame. The origin is the same as that of the $n$-Frame. The $X_{b}$ axis is identical to the body axis.

Next, symbol definitions are described. A general column vector is expressed as $\underline{x}$, a three-dimensional vector is expressed as $\vec{x}$, and a quaternion is expressed as $\tilde{q}$ or $\left\{\begin{array}{c}q_{0} \\ \vec{q}\end{array}\right\}$ where $q_{0}$ and $\vec{q}$ are the scalar element and the vector element, respectively. $\tilde{q}^{*} \equiv\left\{\begin{array}{c}q_{0} \\ -\vec{q}\end{array}\right\}$ indicates the conjugate quaternion of $\tilde{q}$. In addition, we introduce the following superscripts and subscripts: $x_{1}^{2}$ stands for the value of the 1 -Frame coordinatized in the 2-Frame, and $x_{1 / 2}^{3}$ stands for the rotation value of the 1 -Frame relative to the 2 -Frame coordinatized in the 3-Frame. 


\subsection{Extended Kalman filter}

The method of integrating our INS and GPS plays a very important role, because the precision of the INS with MEMS sensors is very low, as previously noted, and we have to utilize another system, a GPS, as much as possible. Therefore, we developed an INS/GPS fusion algorithm based on the extended Kalman filter (EKF) that enables us to perform optimal estimation based on probability. In this subsection, we introduce the general EKF, and describe how the EKF is applied to our INS/GPS.

The EKF is based on two equations: a system equation and measurement equation. The system equation is:

$$
\underline{x}_{t+1}=f\left(\underline{x}_{t}, \underline{u}_{t}\right),
$$

where $\underline{x}_{t}$ and $\underline{u}_{t}$ represent the system's true state values and true inputs, respectively. The measurement equation is:

$$
\underline{z}_{t}=h\left(\underline{x}_{t}\right)+\underline{v}_{t},
$$

where $\underline{z}_{t}$ and $\underline{v}_{t}$ represent measurement values and measurement error, respectively.

In practical applications, the EKF is divided into two stages: time update and measurement update. The time update is performed as time passes and is composed of the following equations:

$$
\begin{aligned}
& \hat{\underline{x}}_{t+1}=f\left(\underline{\hat{x}}_{t}, \hat{\underline{u}}_{t}\right) \\
& P_{t+1}=\Gamma_{t} P_{t} \Gamma_{t}^{\mathrm{T}}+\Phi_{t} Q_{t} \Phi_{t}^{\mathrm{T}} .
\end{aligned}
$$

The measurement update is performed when additional information is gained, and consists of the following equations:

$$
\begin{aligned}
K_{t} & =P_{t} H_{t}^{\mathrm{T}}\left(H_{t} P_{t} H_{t}^{\mathrm{T}}+R_{t}\right)^{-1} \\
\underline{\hat{x}}_{t} & \leftarrow \underline{\hat{x}}_{t}+K_{t}\left(\underline{z}_{t}-h_{t}\left(\underline{\hat{x}}_{t}\right)\right) \\
P_{t} & \leftarrow\left(I-K_{t} H_{t}\right) P_{t} .
\end{aligned}
$$

In these equations, $\underline{\hat{x}}_{t}, \underline{\hat{\underline{u}}}_{t}$ represent the system's estimated state values and estimated inputs, respectively. $P_{t}, Q_{t}$, and $R_{t}$ are error covariance matrices of the system's state residuals between true values and estimated values, input residuals between true values and estimated values, and measurement error, respectively:

$$
\begin{aligned}
& P_{t}=E\left[\left(\underline{\hat{x}}_{t}-\underline{x}_{t}\right)\left(\underline{\hat{x}}_{t}-\underline{x}_{t}\right)^{\mathrm{T}}\right] \equiv\left[\Delta \underline{x}_{t} \Delta \underline{x}_{t}^{\mathrm{T}}\right] \\
& Q_{t}=E\left[\left(\underline{\hat{\hat{u}}}_{t}-\underline{u}_{t}\right)\left(\underline{\hat{u}}_{t}-\underline{u}_{t}\right)^{\mathrm{T}}\right] \equiv\left[\Delta \underline{u}_{t} \Delta \underline{u}_{t}^{\mathrm{T}}\right] \\
& R_{t}=E\left[\underline{v}_{t} \underline{v}_{t}^{\mathrm{T}}\right],
\end{aligned}
$$

where $\Gamma_{t}, \Phi_{t}$ and $H_{t}$ are matrices derived from the perturbation (linearization) forms of the system equation and measurement equation that are nonlinear. In the standard EKF, these matrices are Jacobian:

$$
\begin{aligned}
\Gamma_{t} & \left.\equiv \frac{\partial f}{\partial x}\right|_{x_{t}} \\
\Phi_{t} & \left.\equiv \frac{\partial f}{\partial w}\right|_{x_{t}} \\
H_{t} & \left.\equiv \frac{\partial h}{\partial x}\right|_{x_{t}} .
\end{aligned}
$$

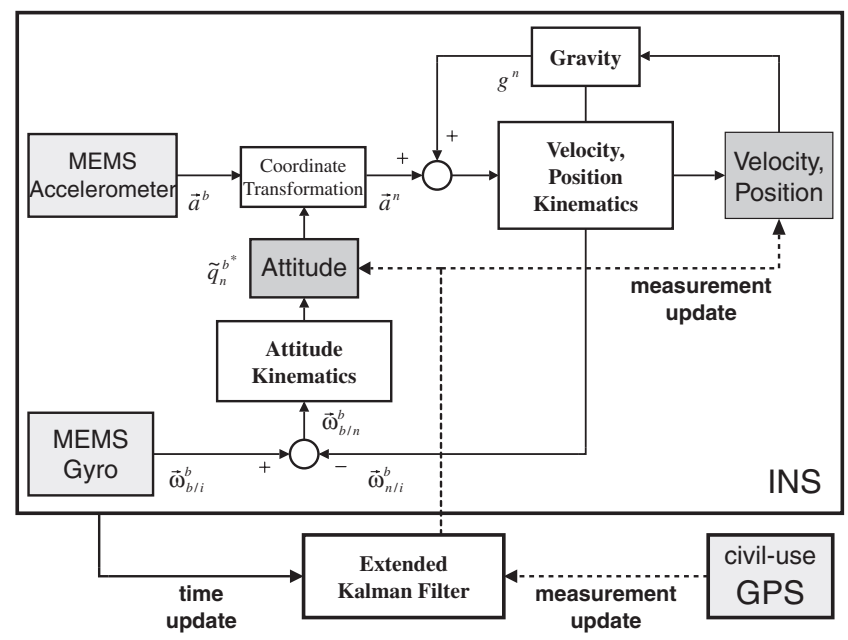

Fig. 2. INS/GPS algorithm.

$K_{t}$ is a matrix called Kalman gain.

Three well-known methods are commonly used to integrate an INS and a GPS with the EKF: loose-coupling, tight-coupling, and ultra tight-coupling. Among these methods, we choose loose-coupling because this method requires the least calculation power and it is most suitable for general-purpose use. In the loose-coupling method, the system equation of the EKF is the kinematics equations of an INS, and the measurement equation is based on the outputs of a GPS. Expressed in another way, the position, velocity, and attitude of the INS are corrected by the position and velocity obtained from the GPS. Figure 2 shows our INS/GPS configuration integrated by the loose-coupling method.

In the next two subsections, we explain the system equation and measurement equation.

\subsection{System equation (INS)}

The system equation, Eq. (1), is the kinematics equation of an INS. Thus, the system's state values $\underline{x}_{t}$ are the position, velocity, and attitude of an INS. Here, we express position and attitude in unit quaternions to remove singular points. This is in order to avoid the problem of the model error increasing and the outputs deteriorating near a singular point. Especially, during calculation, an INS with MEMS sensors occasionally goes through an unpredictable path in the modeled space because of the large size of the input's noise element. Therefore, we consider this an effective way of improving our system's precision. With quaternions, the kinematics equations are as follows:

- Velocity kinematics

$$
\begin{aligned}
\frac{d}{d t}\left\{\begin{array}{c}
0 \\
\dot{\vec{r}}_{e}^{n}
\end{array}\right\}= & \tilde{q}_{b}^{n *}\left\{\begin{array}{c}
0 \\
\vec{a}^{b}
\end{array}\right\} \tilde{q}_{b}^{n}+\left\{\begin{array}{c}
0 \\
\vec{g}^{n}
\end{array}\right\} \\
& -\left\{\begin{array}{c}
0 \\
\left(2 \vec{\omega}_{e / i}^{n}+\vec{\omega}_{n / e}^{n}\right) \times \dot{\vec{r}}_{e}^{n}
\end{array}\right\} \\
& -\tilde{q}_{e}^{n *}\left\{\begin{array}{c}
0 \\
\vec{\omega}_{e / i}^{e} \times\left(\vec{\omega}_{e / i}^{e} \times \vec{r}_{e}\right)
\end{array}\right\} \tilde{q}_{e}^{n}
\end{aligned}
$$


- Position kinematics

$$
\frac{\mathrm{d}}{\mathrm{d} t} \tilde{q}_{e}^{n}=\frac{1}{2} \tilde{q}_{e}^{n}\left\{\begin{array}{c}
0 \\
\vec{\omega}_{n / e}^{n}
\end{array}\right\}, \quad \frac{\mathrm{d}}{\mathrm{d} t} h=-\left(\dot{r}_{e}^{n}\right)_{Z}
$$

- Attitude kinematics

$$
\begin{aligned}
& \frac{\mathrm{d}}{\mathrm{d} t} \tilde{q}_{n}^{b}=\frac{1}{2}\left[\tilde{q}_{n}^{b}\left\{\begin{array}{c}
0 \\
\vec{\omega}_{b / i}^{b}
\end{array}\right\}\right. \\
& \left.-\left(\left\{\begin{array}{c}
0 \\
\vec{\omega}_{e / i}^{n}
\end{array}\right\}+\left\{\begin{array}{c}
0 \\
\vec{\omega}_{n / e}^{n}
\end{array}\right\}\right) \tilde{q}_{n}^{b}\right]
\end{aligned}
$$

We can combine these equations to:

$$
\frac{\mathrm{d}}{\mathrm{d} t}\left[\begin{array}{c}
\dot{\vec{r}}_{e}^{n} \\
\tilde{q}_{e}^{n} \\
h \\
\tilde{q}_{n}^{b}
\end{array}\right]=f^{\prime}\left(\left[\begin{array}{c}
\dot{\vec{r}}_{e}^{n} \\
\tilde{q}_{e}^{n} \\
h \\
\tilde{q}_{n}^{b}
\end{array}\right],\left[\begin{array}{c}
\vec{a}_{b} \\
\vec{\omega}_{b / i}^{b} \\
\vec{g}^{n}
\end{array}\right]\right) .
$$

Discretize this equation by computational time step $\Delta t$ :

$$
\underline{x}_{t+1}=\left(I+\left.f^{\prime}\right|_{\underline{x}_{t}, \underline{u}_{t}} \Delta t\right) x_{t} \quad\left(\equiv f\left(\underline{x}_{t}, \underline{u}_{t}\right)\right)
$$

where

$$
\underline{x}_{t}=\left[\begin{array}{c}
\dot{\vec{r}}_{e}^{n} \\
\tilde{q}_{e}^{n} \\
h \\
\tilde{q}_{n}^{b}
\end{array}\right]_{t}, \quad \underline{u}_{t}=\left[\begin{array}{c}
\vec{a}_{b} \\
\vec{\omega}_{b / i}^{b} \\
\vec{g}^{n}
\end{array}\right]_{t} .
$$

This equation is the system equation, and identical to Eq. (3).

\subsection{Measurement equation (GPS)}

The measurement equation, Eq. (2), is derived by outputs of a GPS:

$$
\underline{z}_{t} \equiv\left[\begin{array}{c}
\dot{\vec{r}}_{e}^{n} \\
\tilde{q}_{e}^{n} \\
h
\end{array}\right]_{\mathrm{GPS} t},
$$

where the subscription GPS stands for the GPS's output. The output of a GPS is velocity $\dot{\vec{r}}_{e}^{n}$ and position $\tilde{q}_{e}^{n}, h$; thus, attitude $\tilde{q}_{n}^{b}$ is dropped from the system state values.

\subsection{INS/GPS algorithm}

In this subsection, we explain our INS/GPS algorithm based on the EKF equations that have been described. We explain the two stages, time update and measurement update, in sequence. We also explain the initialization method and covariance setting.

\subsubsection{Time update}

Time updates are performed as time passes. In this stage, Eq. (3) (i.e., Eq. (18)) and Eq. (4) are performed. Equation (4) requires matrices $\Gamma$ and $\Phi$. In typical EKF applications, these matrices are Jacobians and obtained by Eqs. (11)-(12). However, we obtain the matrices by performing the following steps.

First, we perform the following substitutions to Eqs. (14)-(16), and subtract the original equations from the substituted equations.

$$
\begin{aligned}
& \dot{\vec{r}}_{e}^{n} \leftarrow \dot{\vec{r}}_{e}^{n}+\Delta \dot{\vec{r}}_{e}^{n} \\
& \tilde{q}_{e}^{n} \leftarrow\left\{\begin{array}{c}
1 \\
\Delta \vec{u}_{e}^{n}
\end{array}\right\} \tilde{q}_{e}^{n}, h \rightarrow h+\Delta h \\
& \tilde{q}_{n}^{b} \leftarrow\left\{\begin{array}{c}
1 \\
\Delta \vec{u}_{n}^{b}
\end{array}\right\} \tilde{q}_{n}^{b} \\
& \vec{a}^{b} \leftarrow \vec{a}^{b}+\Delta \vec{a}^{b}, \vec{\omega}_{b / i}^{b} \rightarrow \vec{\omega}_{b / i}^{b}+\Delta \vec{\omega}_{b / i}^{b} \\
& \vec{g}^{n} \leftarrow \vec{g}^{n}+\Delta \vec{g}^{n},
\end{aligned}
$$

where $\Delta$ stands for small error, and multiplications of two or more $\Delta$ values are negligible.

We now get the following perturbation equation:

$$
\frac{\mathrm{d}}{\mathrm{d} t} \Delta \underline{x}=A \Delta \underline{x}+B \Delta \underline{u},
$$

where $\Delta \underline{x}$ and $\Delta \underline{u}$ stand for the system's state values in the perturbation form and the input's error, respectively:

$$
\Delta \underline{x} \equiv\left[\begin{array}{c}
\Delta \dot{\vec{r}}_{n}^{e} \\
\Delta \vec{u}_{e}^{n} \\
\Delta h \\
\Delta \vec{u}_{n}^{b}
\end{array}\right], \quad \Delta \underline{u} \equiv\left[\begin{array}{c}
\Delta \vec{a}^{b} \\
\Delta \vec{\omega}_{b / i}^{b} \\
\Delta \vec{g}^{n}
\end{array}\right],
$$

where $A$ and $B$ are matrices.

Finally, by discretizing Eq. (26), we obtain matrices $\Gamma$ and $\Phi$ :

$$
\Gamma=I+A \Delta t, \quad \Phi=B \Delta t .
$$

We do not use Jacobians because they violate the unity of the quaternion norm. A Jacobian is a so-called additive form, and if we use it the norm of a quaternion $\|\tilde{q}\|$ is:

$$
\begin{aligned}
\|\tilde{q}+\Delta \tilde{q}\|^{2} & =\left(q_{0}+\Delta q_{0}\right)^{2}+\|\vec{q}+\Delta \vec{q}\|^{2} \\
& \approx\left(q_{0}{ }^{2}+\|\vec{q}\|^{2}\right)+2\left(q_{0} \Delta q_{0}+\vec{q} \cdot \Delta \vec{q}\right) \\
& =1+2\left(q_{0} \Delta q_{0}+\vec{q} \cdot \Delta \vec{q}\right) \neq 1,
\end{aligned}
$$

and the unity of the quaternion, $\|\tilde{q}+\Delta \tilde{q}\|=1$, is broken.

Therefore, we employ the multiplicative form described by Ude. ${ }^{4)}$ A small residual element $\Delta \vec{u}$ where $\|\Delta \vec{u}\| \approx 0$ is introduced, and using it, we define the quaternion perturbation form as:

$$
\tilde{q}+\Delta \tilde{q} \equiv\left\{\begin{array}{c}
1 \\
\Delta \vec{u}
\end{array}\right\} \tilde{q}=\left\{\begin{array}{c}
q_{0}-\Delta \vec{u} \cdot \vec{q} \\
\vec{q}+q_{0} \Delta \vec{u}+\Delta \vec{u} \times \vec{q}
\end{array}\right\},
$$

where the norm is

$$
\begin{aligned}
\|\tilde{q}+\Delta \tilde{q}\|^{2} \equiv & \left(q_{0}-\Delta \vec{u} \cdot \vec{q}\right)^{2} \\
& +\left\|\vec{q}+q_{0} \Delta \vec{u}+\Delta \vec{u} \times \vec{q}\right\|^{2} \\
\approx & \left(q_{0}^{2}-2 q_{0} \Delta \vec{u} \cdot \vec{q}\right) \\
& +\left(\|\vec{q}\|^{2}+2 q_{0} \Delta \vec{u} \cdot \vec{q}\right) \\
= & q_{0}^{2}+\|\vec{q}\|^{2}=1,
\end{aligned}
$$

and the unity of the quaternion is kept.

Thus, while the system model is represented by 12 state 
values, $\dot{\vec{r}}_{e}^{n}, \tilde{q}_{e}^{n}, h$, and $\tilde{q}_{n}^{b}$, the perturbation of the system equation is represented by 10 state values, $\Delta \dot{\vec{r}}_{e}^{n}, \Delta \overrightarrow{\vec{u}}_{e}^{n}, \Delta h$, and $\Delta \vec{u}_{n}^{b}$, and the error covariance matrices, $P \equiv E\left[\Delta \underline{x} \Delta \underline{x}^{\mathrm{T}}\right]$, $Q \equiv E\left[\Delta \underline{u} \Delta \underline{u}^{\mathrm{T}}\right]$ are $10 \times 10$ and $9 \times 9$, respectively.

\subsubsection{Measurement update}

When the output of the GPS is available, measurement updates are performed. At this stage, Eqs. (5)-(7) are activated. However, since we use 10 state values, $\Delta \dot{\vec{r}}_{e}^{n}, \Delta \vec{u}_{e}^{n}$, $\Delta h$, and $\Delta \vec{u}_{n}^{b}$, in the perturbation form of the system equation, we have to change the standard equations for the measurement update. This is because there now arises certain problems; for example, the error covariance matrix $P$ is a $10 \times 10$ matrix and the Jacobian $H$ matrix derived from Eq. (13) is $8 \times 12$. Thus, Eq. (5) is broken.

Therefore, we use the following equations instead:

$$
\begin{aligned}
\underline{z}_{t}-h_{t}\left(\underline{\hat{x}}_{t}\right) \equiv-\left[\begin{array}{cccc}
I & 0 & 0 & 0 \\
0 & I & 0 & 0 \\
0 & 0 & I & 0
\end{array}\right]\left[\begin{array}{c}
\Delta \dot{\vec{r}}_{e}^{n} \\
\Delta \tilde{q}_{e}^{n} \\
\Delta h \\
\Delta \tilde{q}_{n}^{b}
\end{array}\right]_{t}+\underline{v} \\
=-\left[\begin{array}{cccc}
I & 0 & 0 & 0 \\
0 & H_{22} & 0 & 0 \\
0 & 0 & I & 0
\end{array}\right]\left[\begin{array}{c}
\Delta \dot{\vec{r}}_{e}^{n} \\
\Delta \vec{u}_{e}^{n} \\
\Delta h \\
\Delta \vec{u}_{n}^{b}
\end{array}\right]_{t}+\underline{v} \\
\equiv H_{t} \Delta \underline{x}_{t}+\underline{v}, \quad \\
H_{22} \equiv\left[\begin{array}{ccc}
-q_{1} & -q_{2} & -q_{3} \\
q_{0} & q_{3} & -q_{2} \\
-q_{3} & q_{0} & q_{1} \\
q_{2} & -q_{1} & q_{0}
\end{array}\right]_{\hat{\tilde{q}}_{e}^{n}}
\end{aligned}
$$

where matrix $H_{t}$ is $7 \times 10$, and we can calculate the Kalman gain $K_{t}$ from Eq. (5), and correct the error covariance matrix $P$ using Eq. (7). Moreover, the system's estimated state values are corrected by the following equations:

$$
\begin{aligned}
& \Delta \underline{\hat{x}}_{t} \equiv\left[\begin{array}{r}
\Delta \hat{\overrightarrow{\vec{r}}}_{n}^{e} \\
\Delta \hat{\vec{u}}_{e}^{n} \\
\Delta \hat{\hat{h}} \\
\Delta \hat{\vec{u}}_{n}^{b}
\end{array}\right]_{t}=K_{t}\left(\underline{z}_{t}-h\left(\underline{\hat{x}}_{t}\right)\right) \\
& \left(\hat{\overrightarrow{\vec{r}}}_{n}^{e}\right)_{t} \leftarrow\left(\hat{\dot{\vec{r}}}_{n}^{e}\right)_{t}-\left(\Delta \hat{\overrightarrow{\vec{r}}}_{n}^{e}\right)_{t} \\
& \left(\hat{\tilde{q}}_{e}^{n}\right)_{t} \leftarrow\left\{\begin{array}{c}
1 \\
\left(\Delta \hat{\vec{u}}_{e}^{n}\right)_{t}
\end{array}\right\} *\left(\hat{\tilde{q}}_{e}^{n}\right), \quad \hat{h}_{t} \leftarrow \hat{h}_{t}-(\Delta \hat{h})_{t} \\
& \left(\hat{\tilde{\boldsymbol{q}}}_{n}^{b}\right)_{t} \leftarrow\left\{\begin{array}{c}
1 \\
\left(\Delta \hat{\overrightarrow{\boldsymbol{u}}}_{n}^{b}\right)_{t}
\end{array}\right\} *\left(\hat{\tilde{\boldsymbol{q}}}_{n}^{b}\right)
\end{aligned}
$$

where Eqs. (34)-(37) are an alternative form of Eq. (6). 3.5.3. Initialization method and covariance setting

Initialization is performed in the quiescent condition. The initial velocity $\hat{\vec{r}}_{e}^{n}$ and position $\hat{\tilde{q}}_{e}^{n}, \hat{h}$ of the system equation
Table 1. Comparison of the covariance $P_{\Delta \vec{u}_{n}^{b} x}$.

\begin{tabular}{lccc}
\hline \multicolumn{1}{c}{ Initial latitude [deg] } & 0 & 40 & 80 \\
\hline True & $2.16 \times 10^{-5}$ & $2.16 \times 10^{-5}$ & $2.17 \times 10^{-5}$ \\
Our algorithm & $2.13 \times 10^{-5}$ & $2.13 \times 10^{-5}$ & $2.13 \times 10^{-5}$ \\
Conventional algorithm & $2.13 \times 10^{-5}$ & $25.7 \times 10^{-5}$ & $62.7 \times 10^{-5}$ \\
\hline
\end{tabular}

are the output values from a GPS receiver at the starting time of measurement. The attitude $\hat{\tilde{q}}_{n}^{b}$ is initialized by sensing the gravity vector and assuming the heading of the target is 0 , because our prototype has no augmentation system such as an Earth magnetism sensor, which senses the absolute heading of the target.

The initial $P_{t}$ covariance matrix of the EKF is a suitably large diagonal matrix. The $Q_{t}$ and $R_{t}$ covariance matrices are determined, respectively, by the measured static noise of inertial sensors and by the dilution of precision (DOP) values acquired from a GPS receiver.

\subsection{Performance analysis}

The algorithm we developed can predict the real model more accurately than conventional algorithms that use Euler angles. We show this performance based on the results of a simulation.

In the simulation, we use two algorithms. One is the developed algorithm, and the other is the conventional one, which uses Euler angles in position (i.e., latitude, longitude, and azimuth). We compare the propagation of the system covariance matrix $P$ of the two algorithms after the iteration of time updates for $300 \mathrm{sec}$. We analyze three cases whose initial latitudes are 0,40 , and 80 degrees, respectively. The other conditions are the same for all cases: 1) the initial state values are true values, and all are 0 except for latitude, 2) the inertial sensor inputs, i.e., $\vec{a}^{b}, \vec{\omega}_{b / i}^{b}$, are only composed of the white noise whose intensity is determined by measuring real MEMS sensors. In addition, we obtain the true system covariance by performing the simulation 1,000 times.

Table 1 shows one of the results, which is $P_{\Delta \vec{u}_{n}^{b} x}$, the true and predicted covariances of the first element of the attitude error $\Delta \vec{u}_{n}^{b}$. According to the results, in the lower latitude, both algorithms can predict the covariances correctly, but in the higher latitude, the conventional algorithm fails. This reflects the growth of the modeling error by the singular point of the Euler angle that is located at 90 degrees. Therefore, our algorithm decreases the possibility of divergence by reducing the error between computational models and real models.

\section{Evaluation}

In order to evaluate our suggested configuration, we constructed a prototype system. Moreover, after calibration, we performed an experiment comparing the prototype with GAIA, a high-precision INS/GPS device developed by JAXA. In this section, we describe the details of the prototype, its calibration and the experiment results.

\subsection{Prototype}

Figures 3 and 4 show a photograph and a processing flow 


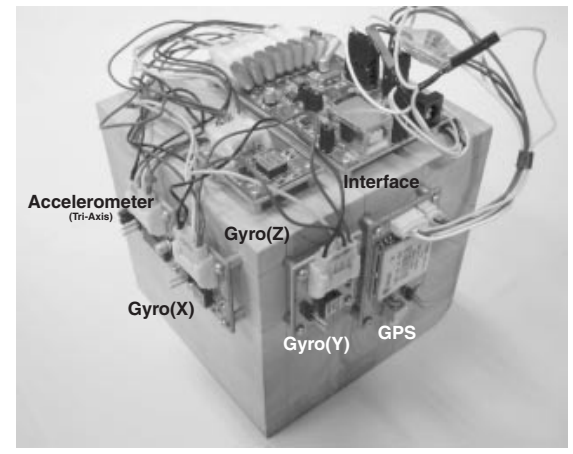

Fig. 3. Photograph of the prototype.

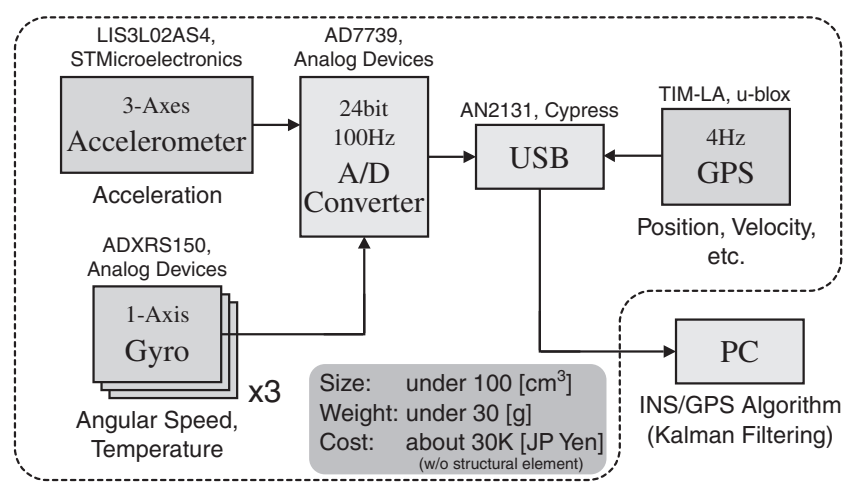

Fig. 4. Block diagram of the prototype.

diagram of the prototype system, respectively. The prototype consists of MEMS inertial sensors, a GPS receiver, an interface circuit, and a personal computer (PC). The MEMS inertial sensors are an accelerometer made by STMicroelectronics and gyros made by Analog Devices, and their data (i.e., acceleration and angular speed) are collected at $100 \mathrm{~Hz}$. The GPS receiver is a GPS module made by u-blox, and its data (i.e., position and velocity) are read out at $4 \mathrm{~Hz}$. In other words, the time updates and the measurement updates of the INS/GPS algorithm are performed at $100 \mathrm{~Hz}$ and $4 \mathrm{~Hz}$, respectively. We also record the temperature sensed by the gyros for temperature calibration, as will be described later. A PC is used to apply the INS/GPS algorithm to the collected raw data. For simplicity, we gather the raw data in real-time and subsequently perform the calculations. We also have confirmed that the PC is exchangeable for a small digital signal processor (DSP) unit, which have enough calculation power for real-time application.

The size, weight, and material cost of the prototype are under $100 \mathrm{~cm}^{3}$, under $30 \mathrm{~g}$ and about US\$300 without the $\mathrm{PC}$ and structural elements. Our proposed configuration is small, light, and inexpensive enough for general-purpose usage.

\subsection{Calibration}

Before the experiment, we measured the bias temperature characteristics and the misalignment of the inertial sensors, and canceled these effects by numerical calibration. We chose to correct these two aspects of the system because they are considered to be the major causes of the degrada-

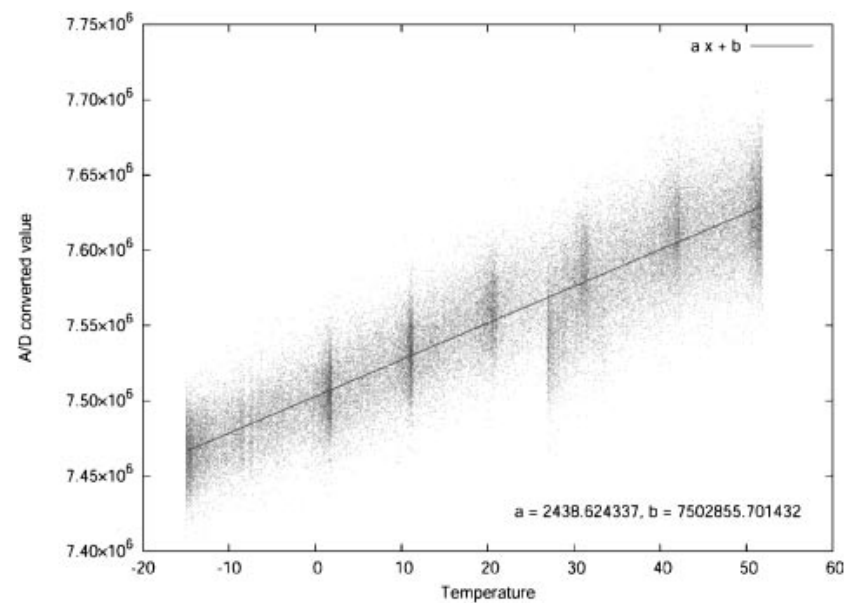

Fig. 5. Temperature characteristics of the $X$-axis gyro bias.

Table 2. List of temperature characteristics.

\begin{tabular}{cccc}
\hline Sensor type & $X$-axis & $Y$-axis & $Z$-axis \\
\hline $\begin{array}{c}\text { Accelerometer } \\
{\left[\mathrm{m} / \mathrm{sec}^{2} /{ }^{\circ} \mathrm{C}\right]}\end{array}$ & -0.000763 & 0.0223 & 0.00602 \\
$\mathrm{Gyro}$ & & & \\
{$\left[\mathrm{deg} / \mathrm{sec} /{ }^{\circ} \mathrm{C}\right]$} & 0.0575 & -0.0881 & -0.0191 \\
\hline
\end{tabular}

tion of the system's precision. First, it is noted that MEMS inertial sensors are intended for detecting large displacements and their bias stability in relation to changes in temperature is very low. In addition, inertial sensors have to be aligned orthogonally as precisely as possible. We describe the details in the following.

\subsubsection{Relation of bias and temperature}

The bias temperature characteristics of accelerometers and gyros are obtained by setting them statically in a thermostatic chamber at a controlled temperature. If the sensor's bias is affected by operating temperature, the results revealed by a graph of sensed value versus temperature will be uneven. Part of the measured data shown in Fig. 5 demonstrates a liner relationship between temperature and bias. Table 2 shows the results of analysis in which the biases of all the sensors are sensitive to changes in operating temperature.

\subsubsection{Misalignment}

The misalignment of the prototype's gyros is measured by collecting the output of the prototype fixed on a turntable which rotates at a constant speed and analyzing this data. Figure 6 shows a photograph of the prototype and the turning table.

We compute the misalignment of gyros by the following scheme. First, we introduce some notations. We describe an unit vector on the rotation detector, and the scale factor of the $X$-axis gyro as $\vec{u}_{x}$ and $K_{x}$, respectively. A unit vector that is orthogonal to an attachment surface signified by $\mathrm{A}$ is described as $\vec{u}_{0 \mathrm{~A}}$. The output of the $X$-axis gyro when surface $\mathrm{A}$ is the reference surface is expressed as $\vec{a}_{x \mathrm{~A}}$.

Then, when we measure outputs relative to surface A, B, and $\mathrm{C}$, there is the following relation: 


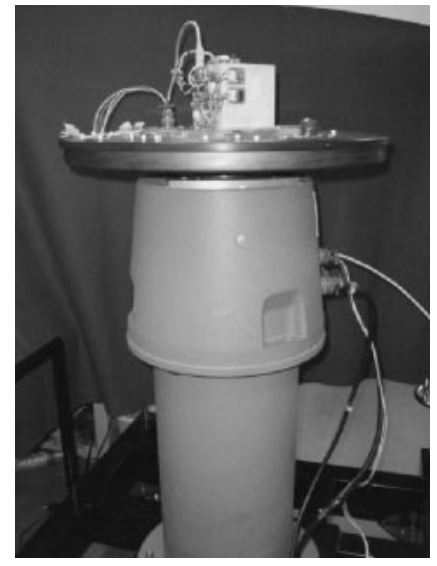

Fig. 6. Misalignment measurement.

Table 3. Gyro misalignment.

\begin{tabular}{cccc}
\hline & $X$-axis & $Y$-axis & $Z$-axis \\
\hline$\vec{u}$ & 1.00 & $8.72 \times 10^{-3}$ & $-1.18 \times 10^{-2}$ \\
& $-9.43 \times 10^{-3}$ & 1.00 & $4.91 \times 10^{-3}$ \\
& $1.52 \times 10^{-3}$ & $1.24 \times 10^{-2}$ & 1.00 \\
\hline $\begin{array}{c}\text { Misalignment } \\
{[\mathrm{deg}]}\end{array}$ & 0.547 & 0.868 & 0.731 \\
\hline
\end{tabular}

$$
\left[\begin{array}{ccc}
\frac{\vec{a}_{x \mathrm{~A}}}{K_{x}} & \frac{\vec{a}_{y \mathrm{~A}}}{K_{y}} & \frac{\vec{a}_{z \mathrm{~A}}}{K_{z}} \\
\frac{\vec{a}_{x \mathrm{~B}}}{K_{x}} & \frac{\vec{a}_{y \mathrm{~B}}}{K_{y}} & \frac{\vec{a}_{z \mathrm{~B}}}{K_{z}} \\
\frac{\vec{a}_{x \mathrm{C}}}{K_{x}} & \frac{\vec{a}_{y \mathrm{C}}}{K_{y}} & \frac{\vec{a}_{z \mathrm{C}}}{K_{z}}
\end{array}\right]=\left[\begin{array}{l}
\vec{u}_{0 \mathrm{~A}}^{\mathrm{T}} \\
\vec{u}_{0 \mathrm{~B}}^{\mathrm{T}} \\
\vec{u}_{0 \mathrm{C}}^{\mathrm{T}}
\end{array}\right]\left[\begin{array}{lll}
\vec{u}_{x} & \vec{u}_{y} & \vec{u}_{z}
\end{array}\right] .
$$

Using this equation, where $\vec{u}_{0 \mathrm{~A}}$, etc. are the known normal vectors of surfaces the gyros are attached to, we can compute the misalignment $\vec{u}_{x}$, etc. and the scale factor $K_{x}$ using the unit condition $\left\|\vec{u}_{x}\right\|=\left\|\vec{u}_{y}\right\|=\left\|\vec{u}_{z}\right\|=1$.

The results are shown in Table 3. Measuring the misalignment of the prototype's accelerometer is omitted because it contains three axes in a single package and is factory calibrated.

\subsection{Experiment}

We compared the output of the prototype with that of GAIA in order to evaluate the precision of the prototype. GAIA is a high-precision INS/GPS device with an absolute position error of under $1 \mathrm{~m}$, which is sufficiently reliable as a reference. The experiment was carried out during the flight of an experimental aircraft, MuPAL- $\alpha$ owned by JAXA, and horizontal straight flight, steady turning flight, and so on were conducted with both the prototype system and GAIA installed.

Figures 7-9 respectively show a comparison of position, velocity, and attitude between the prototype and GAIA. In the results, the difference of mounting positions is considered and canceled. We can recognize that the prototype's output is almost equal to that of GAIA. Additionally, Table 4 shows a statistical summary of the results. This summary is computed based on values from the time that

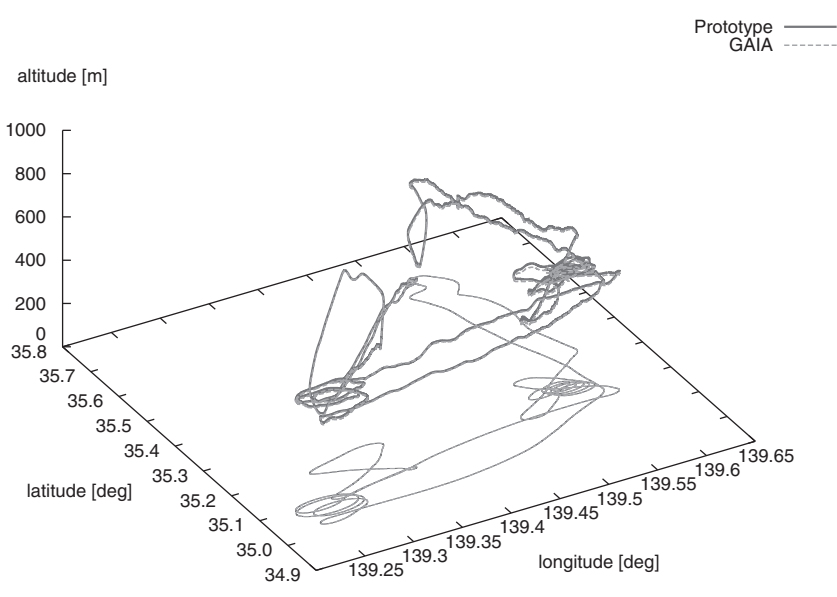

Fig. 7. Position history.
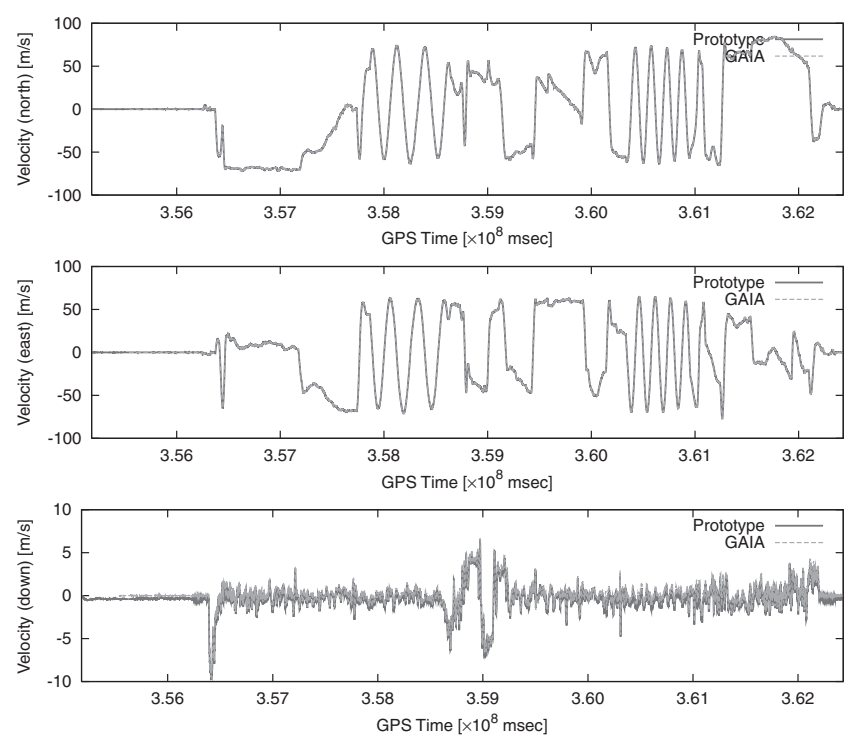

Fig. 8. Velocity history.
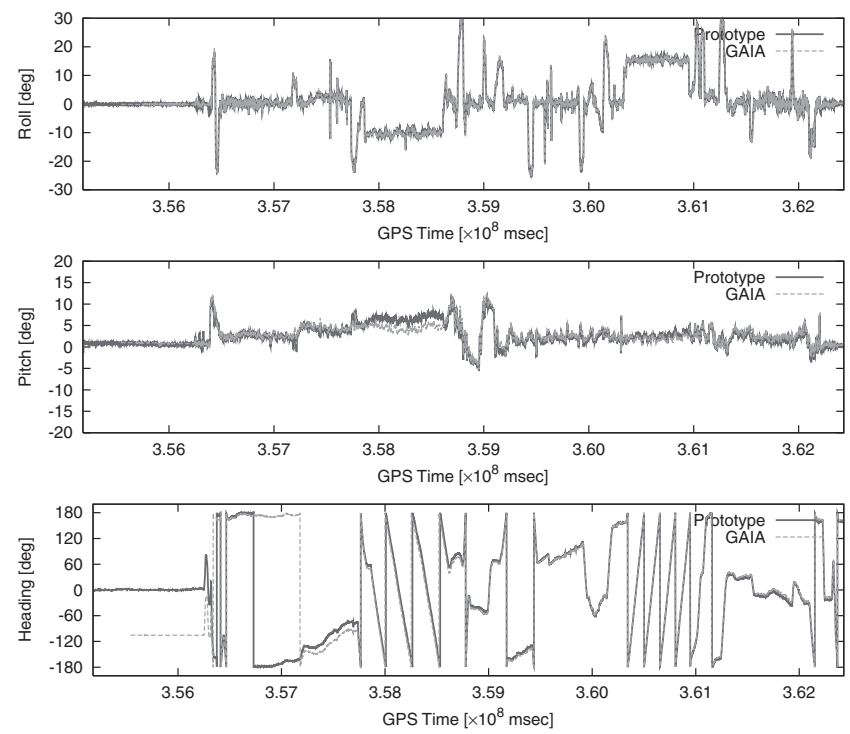

Fig. 9. Attitude history. 
Table 4. Difference between the results of the prototype and those of GAIA.

\begin{tabular}{lccc}
\hline & Mean & $\begin{array}{c}\text { Standard } \\
\text { deviation }\end{array}$ & Worst \\
\hline Horizontal distance $[\mathrm{m}]$ & 6.44 & 2.97 & 17.0 \\
Altitude [m] & 0.85 & 2.10 & 6.90 \\
North speed [m/sec] & 0.00 & 0.12 & 1.25 \\
East speed [m/sec] & 0.00 & 0.12 & -1.13 \\
Down speed [m/sec] & -0.08 & 0.10 & -0.67 \\
Roll [deg] & 0.00 & 0.26 & -1.19 \\
Pitch [deg] & -0.67 & 1.21 & -3.90 \\
Heading [deg] & 4.17 & 9.68 & 23.9 \\
\hline
\end{tabular}
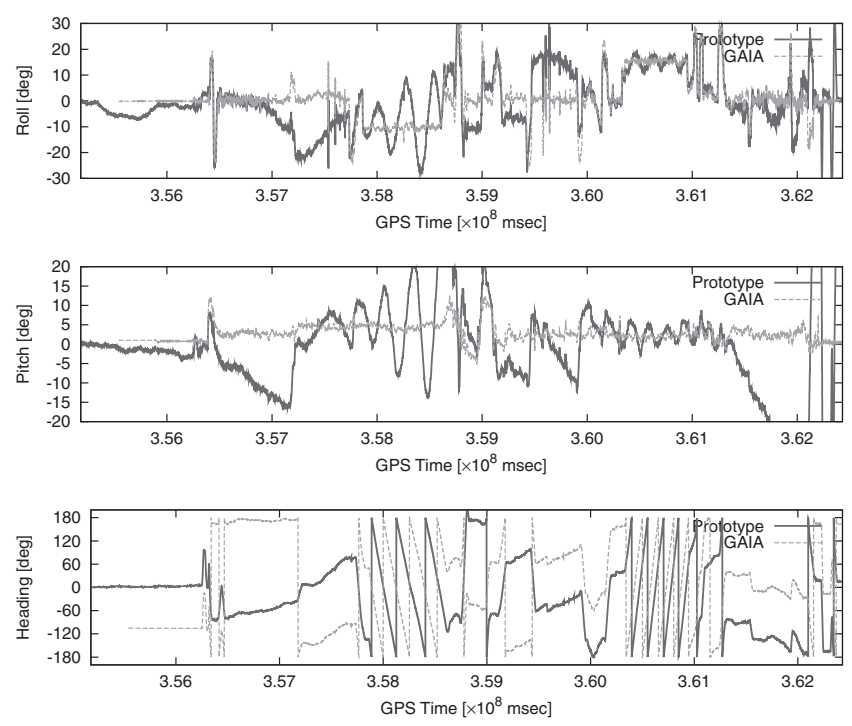

Fig. 10. Attitude history (prototype INS).

the error covariance matrix $P$ of the EKF has sufficiently converged (i.e., at the GPS time of $3.57 \times 10^{8} \mathrm{~ms}$ ). According to the statistical summary, in consideration of the mean and standard deviations, the precision achieved by the prototype is under $10 \mathrm{~m}$ in regard to position, about $1 \mathrm{~m} / \mathrm{s}$ in velocity, under 2 degrees in roll and pitch, but more than 10 degrees in heading.

\subsection{Discussion}

According to the experimental results, the error margin of our system is about several meters in regard to position and more than ten degrees regarding heading, which is the area of lowest performance in consideration of standard deviation. We conclude that our configured INS/GPS is sufficiently precise for the general-purpose control and monitoring of moving objects.

In addition, the results show that we have built an effective navigation system through integration of a MEMS INS, whose precision is very poor when used alone, and a GPS. We focus on attitude in order to support this discussion, because attitude is not directly observed by a GPS receiver. Figure 10 shows the attitude history of the prototype INS (i.e., the system that is configured by only MEMS inertial sensors mounted on the prototype). The attitude history of the prototype INS is not accurate, but rather diverged.

When looking closely at the experimental results, we notice that the accuracy of heading information is much worse than information regrading roll and pitch. We consider this a reflection of the poor performance of the MEMS gyros. That is to say, roll and pitch can be compensated by the Earth's gravitation obtained by accelerometers, because it is much larger than the target's acceleration derived from the movement. Meanwhile, absolute heading cannot be augmented by acceleration, but only by the Earth's rotation rate, which is very small and cannot be measured with low $\mathrm{S} / \mathrm{N}$ gyros such as a MEMS gyro. Thus, the precision of heading is most reflected in the gyro's performance, and we conclude that the deterioration of precision is especially derived from the MEMS gyros.

Based on the above discussion, in order to increase the precision of the suggested system, we have two approaches. One is the hardware approach. It is worthwhile to integrate another aiding system that compensates for attitude, which is related to the performance of gyros. For example, a magnetic compass is available. The other is the software approach. If we analyze the MEMS gyro's error and obtain a more detailed mathematical model, the system performance may be improved.

\section{Conclusion}

We have proposed a new INS/GPS fusion algorithm based on quaternions, and have proven through simulations that the algorithm performance is superior to the conventional algorithm, which uses Euler angles in position. We have also shown that the proposed prototype INS/GPS configuration is sufficiently small, light weight and inexpensive for general-purpose usage. As well, the flight experiments indicated that our INS/GPS configuration has a position error within a few meters and under 2 degrees of roll and pitch error, which is sufficiently precise for controlling and monitoring moving objects.

Further clues are presented for improving the system's precision. According to the experimental results, a relationship apparently exists between system accuracy and the MEMS gyro's performance. In a future study, we will analyze the relationship.

\section{Acknowledgments}

It is noted that the flight tests using MuPAL- $\alpha$ were carried out in collaboration with JAXA. We would also like to thank Mitsubishi Electric Co., Ltd. for lending the instruments used for calibration.

\section{References}

1) Winkler, S., Buschmann, M., Kruger, L., Schulz, H. and Vörsmann, P.: State Estimation by Multi-Sensor Fusion for Autonomous Mini and Micro Aerial Vehicles, Paper 2005-5840 at AIAA Guidance, Navigation, and Control Conference and Exhibit, 2005.

2) Liu, J., Li, R., Niu, X. and Qiao, L.: MEMS-Based Inertial Integrated 
Navigation Technology for Micro Air Vehicles, Paper 2006-6547 at AIAA Guidance, Navigation, and Control Conference and Exhibit, 2006.

3) Harigae, M., Tomita, H. and Nishizawa, T.: Development of High Precision GPS Aided Inertial Navigation, J. Jpn. Soc. Aeronaut. Space Sci., 50 (2002), pp. 416-425 (in Japanese).

4) Ude, A.: Filtering in a Unit Quaternion Space for Model-Based Object Tracking, Robotics and Autonomous Systems, 28 (1999), pp. 163-172.

5) Rogers, R.M.: Applied Mathematics in Integrated Navigation System, Second Edition, AIAA Education Series, ISBN 1-56347-656-8, 2003.

6) Kalman, R.E.: A New Approach to Linear Filtering and Prediction
Problems, Trans. ASME-J. Basic Eng., 82(D), (1960) pp. 35-45.

7) Choukroun, D.: A Novel Quaternion Kalman Filter, Paper 2002-4460 at 42th AIAA Guidance, Navigation, and Control Conference, 2004.

8) Simon, D.: Optimal State Estimation, ISBN 0-471-70858-5, 2006.

9) Katayama, T.: Applied Kalman Filtering, New Edition, ISBN 4-25420101-X, 2000 (in Japanese).

10) Tamagawa Seiki Co., Ltd.: Introduction to Gyro Application Technology, ISBN 4-7693-1208-3, 2003 (in Japanese).

11) Naruoka, M. and Tsuchita, T.: A Low-cost Integrated Navigation System of INS/GPS Using MEMS Sensors, 37th JSASS Annual Conference, 2006 (in Japanese). 